\title{
Big Data Flow Adjustment Using Knapsack Problem
}

\author{
Eyman Yosef ${ }^{1}$, Ahmed Salama ${ }^{1}$, M. Elsayed Wahed ${ }^{2}$ \\ ${ }^{1}$ Department of Mathematics, Faculty of Sience, Bour Said University, Bour Said, Egypt \\ ${ }^{2}$ Faculty of Computers and Informatics, Suez Canal University, Ismailia, Egypt \\ Email: mewahed@yahoo.com
}

How to cite this paper: Yosef, E., Salama, A. and Wahed, M.E. (2018) Big Data Flow Adjustment Using Knapsack Problem. Journal of Computer and Communications, 6, 30-39. https://doi.org/10.4236/jcc.2018.610003

Received: July 3, 2018

Accepted: October 26, 2018

Published: October 29, 2018

Copyright (C) 2018 by authors and Scientific Research Publishing Inc. This work is licensed under the Creative Commons Attribution International License (CC BY 4.0).

http://creativecommons.org/licenses/by/4.0/

\section{(c) (†) Open Access}

\begin{abstract}
The advancements of mobile devices, public networks and the Internet of creature huge amounts of complex data, both construct $\&$ unstructured are being captured in trust to allow organizations to produce better business decisions as data is now pivotal for an organizations success. These enormous amounts of data are referred to as Big Data, which enables a competitive advantage over rivals when processed and analyzed appropriately. However Big Data Analytics has a few concerns including Management of Data, Privacy \& Security, getting optimal path for transport data, and Data Representation. However, the structure of network does not completely match transportation demand, i.e., there still exist a few bottlenecks in the network. This paper presents a new approach to get the optimal path of valuable data movement through a given network based on the knapsack problem. This paper will give value for each piece of data, it depends on the importance of this data (each piece of data defined by two arguments size and value), and the approach tries to find the optimal path from source to destination, a mathematical models are developed to adjust data flows between their shortest paths based on the 0 - 1 knapsack problem. We also take out computational experience using the commercial software Gurobi and a greedy algorithm (GA), respectively. The outcome indicates that the suggest models are active and workable. This paper introduced two different algorithms to study the shortest path problems: the first algorithm studies the shortest path problems when stochastic activates and activities does not depend on weights. The second algorithm studies the shortest path problems depends on weights.
\end{abstract}

\section{Keywords}

0 - 1 Knapsack Problem, Big Data, Big Data Analytics, Big Dao Ta Inconsistencies 


\section{Introduction}

Big data is a big deal. We read about it and its promises of insight. But we will need a network to collect and distribute big data connected to processing locations. Many big data applications require real-time communications. Plan for big data on your network now; don't wait until issues arrive. Catch-up costs money and results in delayed implementations.

The only sure predictions around big data's impact are that the network will be busier, need more capacity, and probably cost more. How much capacity will be needed is only an estimate. It could wind up being far more than estimated if the big data applications are very successful. Educated predictions on traffic may look good now, but conditions can change and render them inaccurately.

Real-time processing of big data will require real-time data delivery; data will already be old and historical. One of the advantages of big data, especially in regard to the Internet of Things (IoT), is its enabling of a rapid response to changing business functions and conditions such as security alerts, building automation, location tracking, etc. Big data collected quickly fosters just-in-time decisions.

The United Nations Economic Commission for Europe predicts that data growth will be $350 \%$ higher in 2019 than it is in 2015; Figure 1 shows the expected growth of size of data in 2019. Such volume of data means a corresponding $350 \%$ growth in network traffic, which may be carried over private LANs (wired and wireless) and WANs, the Internet, and cellular networks.

This paper proposes an applicable method to adjust the optimal path for moving big data between source and destination depending on the size and impotence of this data, let us suppose that we have more data need to distribute through given network, according to the importance or value of each data and

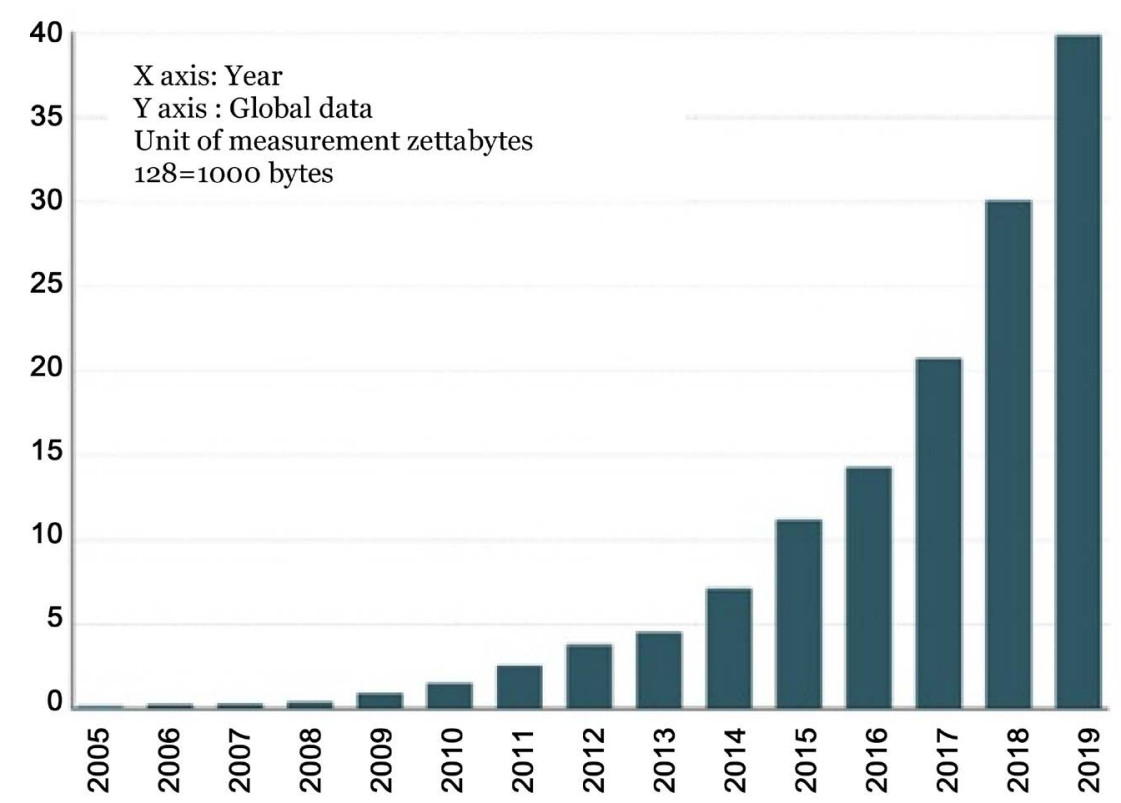

Figure 1. Expected growth size of data. 
total capacity of the network the approach selects the suitable amount of importance data and insure that it is not exceed the total network capacity by using knapsack problem.

\section{Related Work}

The proposed method is concerned with specific topics of resource allocation that have been studied in related literatures. In1996, Yu Gang [1], studied the Max-Min Knapsack (MNK) problem as a NP-hard for an unbounded number of scenarios and pseudo polynomial solvable for a bounded number of scenarios.

The effective of lower and upper bounds were generated by surrogate relaxation. The ratio of these two bounds is shown to be bounded by a constant for situations where the data range is limited to be within a fixed percentage from its mean. A branch-and-bound algorithm has been implemented to efficiently solve the MNK problem to optimality. In 2009, Campegiani and Presti [2], suggested a generalization model of the classical 0/1 Knapsack Problem. They developed a heuristic to obtain very near optimum solutions in a timely manner. In 2013, Zhang et al. [3], proposed a new bio-inspired model to solve problems. The proposed method has three main steps. First, the 0 - 1 knapsack problem is converted into a directed graph by the network converting algorithm. Then, for the purpose of using the amoeboid organism model, the longest path problem is transformed into the shortest path problem. Finally, the shortest path problem can be well handled by the amoeboid organism algorithm. Numerical examples are giving to illustrate the efficiency of the proposed mode. In 2015, Rooderkerk and Heerde [4], developed a robust approach to optimize retail assortments since retailers face the difficult task of designing a portfolio of products that balances risk and return. They proposed a novel, efficient and real-time heuristic depends on 0 - 1 Knapsack that solves the problem and offers an optimal balance. The heuristic constructs an approximation of the risk-return Efficient Frontier of assortments.

\section{Knapsack Problem}

\subsection{The Unbounded Knapsack Problem}

As a typical non-deterministic polynomial-time hard (NP-hard) problem, the unbounded knapsack problem (UKP) is defined as follows: We are given a set of $n$ types $O=\left\{o_{1}, o_{2}, \cdots, o_{n}\right\}$ of items without quantity restriction. Items of the same type share a common weight $w_{i}$ and a common value $\phi_{i}$. The problem is to choose a subset of these items aiming to maximize their overall value, while their overall weight does not exceed a given capacity $c$. Without loss of generality, it should be assumed that all values and weights are positive, all weights are smaller than the capacity $\mathcal{c}$, and the overall weight of all items exceeds $c$. The model of UKP problem can be formulated as follows:

$$
\operatorname{Maximize} \sum_{i=1}^{n} \phi_{i} x_{i},
$$




$$
\begin{gathered}
\text { Constrain } \sum_{i=1}^{n} w_{i} x_{i} \leq c, \\
\forall x_{i} \in Z^{+}, 1 \leq i \leq n
\end{gathered}
$$

where $x_{i}$ represents the number of items of type $o_{i}$ included in the knapsack.

\subsection{Preliminaries}

In this section, the model of $0-1$ knapsack problem and the amoeboid organism are introduced.

\section{Mathematical model of the amoeboid organism}

From the experiments on the amoeboid organism as described in [5], the mechanism of tube formation can be obtained: tubes thicken in a given direction when shuttle streaming of the protoplasm persists in that direction for a certain time. It implies positive feedback between flux and tube thickness, as the conductance of the sol is greater in a thicker channel.

According to the mechanism, two rules describing the changes in the tubular structure of the amoeboid organism are: first, open-ended tubes, which are not connected between the two food sources, are likely to disappear; second, when two or more tubes connect the same two food sources, the longer tube is likely to disappear [6]. With these two rules, a mathematical model for maze solving problems has been constructed.

The variable $Q_{i j}$ is used to express the flux through tube $M_{i j}$ from $N_{i}$ to $N_{j}$. Assuming the flow along the tube as an approximately poiseuille flow, the flux $Q_{i j}$ can be expressed as [7]:

$$
Q_{i j}=\frac{D_{i j}}{L_{i j}}\left(p_{i}-p_{j}\right),
$$

where $p_{i}$ is the pressure at the node $N_{i}, D_{i j}$ is the conductivity of the edge $M_{i j}$.

Assume zero capacity at each node; hence by considering the conservation law of sol the following equation can be obtained see [2]:

$$
\sum Q_{i j}=0,(j \neq 1,2), i=1, \cdots, n
$$

For the source node $N_{1}$ and the sink node $N_{2}$ the following two equations hold

$$
\begin{aligned}
& \sum Q_{i 1}+I_{0}=0, \\
& \sum Q_{i 2}+I_{0}=0,
\end{aligned}
$$

where $I_{0}$ is the flux flowing from the source node. It can be seen that $I_{0}$ is a constant value in this model.

In order to describe such an adaptation of tubular thickness we assume that the conductivity $D_{i j}$ changes over time according to the flux $Q_{i j}$. The following equation for the evolution of $D_{i j}$ can be used

$$
\frac{\mathrm{d}}{\mathrm{d} t} D_{i j}=f\left(\left|Q_{i j}\right|-r D_{i j}\right)
$$


where $r$ is a decay rate of the tube. It can be obtained that the equation implies that the conductivity ends to vanish if there is no flux along the edge, while it is enhanced by the flux. The $f$ is monotonically increasing continuous function $f(0)=0$.

Then the network Poisson equation for the pressure can be obtained from the Equations (4)-(7) as follows [8]:

$$
\sum_{i} \frac{D_{i j}}{L_{i j}}\left(p_{i}-p_{j}\right)=\left\{\begin{array}{cc}
-1 & \text { for } j=1 \\
+1 & \text { for } j=2 \\
0 & O / W
\end{array}\right.
$$

By setting $p_{2}=0$ as a basic pressure level, all $p_{i}$ can be determined by solving Equation (9) and $Q_{i j}$ can also be obtained.

In this paper, it has been obtained that $\mathrm{f}$ is monotonically increasing continuous function satisfying $f(0)=0$ in Equation (8). Therefore, $f(Q)=|Q|$ is used in this paper. With the flux calculated, the conductivity can be derived, where Equation (10) is used instead of Equation (8), adopting the functional form $f(Q)=|Q|$.

$$
\frac{D_{i j}^{n+1}-D_{i j}^{n}}{\delta t}=\left|Q-D_{i j}^{n+1}\right|
$$

\subsection{Problem Description}

Given an acyclic undirected network $G(N, A)$, consisting of a set of nodes $N=\{1,2, \cdots, n\}$ and $m$ undirected arcs $A \in N \times N$. Each arc is denoted by ordered pair $(i, j)$, where $i, j \in N$. The weight of arc $t=\left(v_{i}, v_{j}\right)$ is denoted by a interval data $w=t_{i}=\left[t_{i}^{-}, t_{i}^{+}\right]$. Given two nodes $v_{i}$ and $v_{t}$, assume $P$ is one path from node $v_{i}$ to node $v_{t}$ in the network $G$. The weight of path $P$ is the sum of the arcs' weight in the path and it is stated as $w(p)$. As a result, the shortest path problem can be formulated as follows [9]:

$$
w\left(p_{0}\right)=\min \sum_{p} w(p)
$$

The following equation is defined to convert $\alpha$ interval data into a crisp number [10].

$$
w=\left\{t_{i}\right\}=\alpha * t_{i}^{-}+(1-\alpha) * t_{i}^{+}, 0 \leq \alpha \leq 1
$$

\section{A Single-Processor Machine for the 0 - 1 Knapsack Problem in Dynamic Programming Method [11]}

Dynamic Programming (DP) solves the problem by producing " $f_{1}, f_{2}, \cdots, f_{n}$ ” sequentially. As mentioned in the previous section, $f_{i}(x)$ is a monotone no lessening basic step work. " $f_{i}(x)$ " may be exemplified as the set $S P_{i}$ from claiming rows from the coordination of that phase focuses of " $f_{i}(x)$ ".

The size of the set $S_{i}$, (i.e. $\left|S P_{i}\right|$ ), is not greater than " $C+1$ " and rows should be planned in growing arrangement $x$ while $f_{i}(x)$. The series of sets, " $S P_{0}, S P_{1}, \cdots, S P_{n}$ " is a history of the DP and that should be backtracked through 
"Algorithm 1" to get the solution vector $x$ see [12].

\begin{tabular}{|c|c|}
\hline \multicolumn{2}{|c|}{ 4.1 Algorithm 1- traditional 0-1 Knapsack Algorithm } \\
\hline Input & $W_{\text {item }}:$ Weight of each item , $P_{\text {item }}:$ Profit of each item, $C$ : Capacity \\
\hline Output & $S P$ : array of subproblem to find an optimal solution, $X: 0$ or 1 select item or not \\
\hline Step 1 & $S P_{i j} \leftarrow\{0,0\} \quad \quad / /$ item $:$ number of item $, \mathrm{TN}:$ total number of item \\
\hline Step 2 & $\begin{array}{l}\text { for item }=1 \text { to TN do } \quad / / \text { Capcount: : Capacity count } \\
\text { For Capcount } \leftarrow 0 \text { to } C \text { do } \\
\text { If } W_{\text {item }} \leq C \text { then } \quad / / \mathrm{SP}: \text { subproblem } \\
\left.\text { If } P_{\text {item }}+\mathrm{SPD} \text { [item -1, Capcount }-W_{\text {item }}\right]>\mathrm{SP} \text { [item-1, Capcount] then } \\
\mathrm{SPD}\left[\text { item }-1 \text {, Capcount } \quad \leftarrow P_{\text {item }}+S P\left[i-1, j-W_{\text {item }}\right]\right. \\
\text { Else } \\
\mathrm{SP} \text { [item, Capcount] } \leftarrow \mathrm{SP} \text { [item -1, Capcount] } \quad / / W_{\text {item }}>C \\
\text { Endif } \\
\text { End for } \quad / / \quad \text { Capcount } \\
\text { End for } \quad / / \quad \text { item }\end{array}$ \\
\hline Step 3 & item $\leftarrow \mathrm{TN}$, Capcount $\leftarrow C$ \\
\hline Step 4 & $\begin{array}{l}\text { while item and Capcount }>0 \\
\text { If } S P \text { [item - 1, Capcount }] \neq S P \text { [item, Capcount }] \text { then } \\
\text { item } \leftarrow \text { item-1, Capcount } \leftarrow \text { Capcount }-W_{\text {item }} \\
X_{\text {tem }}=1 \\
\text { else } \\
\text { item } \leftarrow \text { item-1 } \\
X_{\text {tem }}=0 \\
\text { end if } \\
\text { Endwhile }\end{array}$ \\
\hline
\end{tabular}

\section{Numerical Example 1}

In this section, a numerical example is used to show the efficiency of the proposed method.

As can be seen in Figure 2, an example of shortest path problem with interval arcs is shown. The shortest path from node 1 to node 12 is needed to be found,

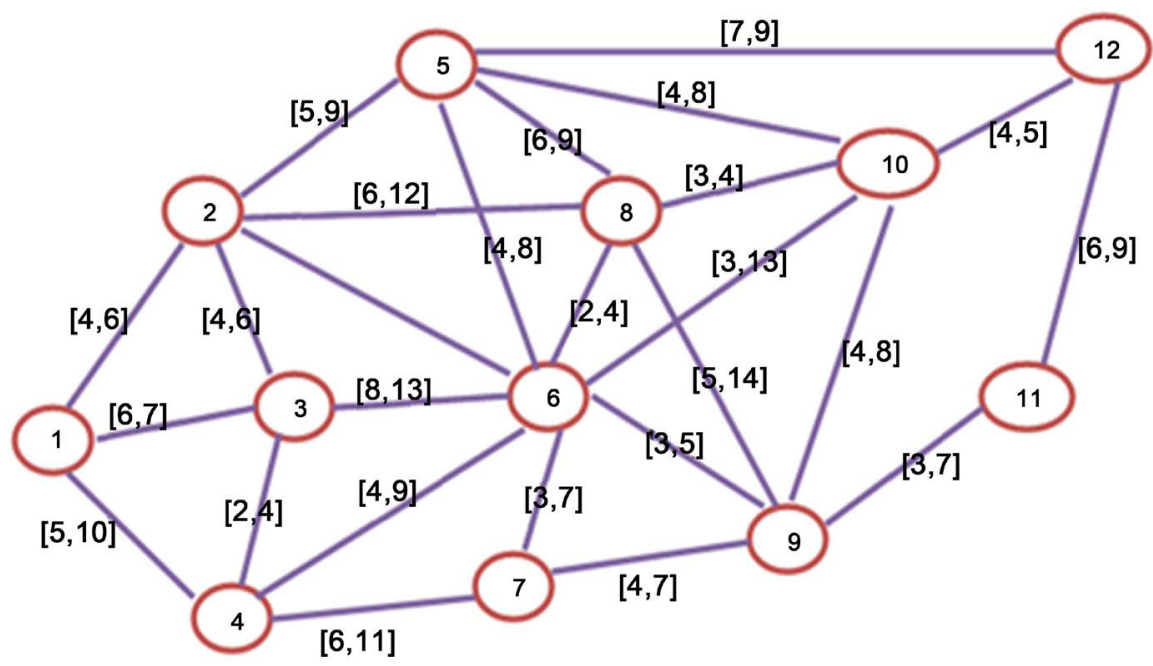

Figure 2. An example of undirected graph with 12 nodes. 
and $0 \leq \alpha \leq 1$, but assume that $\alpha$ is set 0.5 , Figure 3 can be obtained. The matrix corresponding to the converted graph can be obtained as follows.

From Figure 2 we can obtain this Table 1.

From Equation (12) assume all the items' values in the initial conductivity matrix are set $\alpha=0.5$, the shortest path from node 1 to node 12 can be found using the amoeboid organism algorithm and the result in Table 2 obtained from the matlab code program applied on the and Figure 3. It can be obtained that the shortest path is $(1) \rightarrow(2) \rightarrow(6) \rightarrow(8) \rightarrow(10) \rightarrow(12)$. If $\alpha$ is set 1 , the shortest path in the network is $(1) \rightarrow(2) \rightarrow(6) \rightarrow(10) \rightarrow(12)$. If $\alpha$ is set 0 , the shortest path in the network is 1$) \rightarrow(2) \rightarrow(6) \rightarrow(8) \rightarrow(10) \rightarrow(12)$.

It can be seen that different shortest paths are obtained when $\alpha$ has different values.

\section{Numerical Example 2}

Consider the problem $(\mathrm{X})$ with the following given data in Table 1, where $\mathrm{n}$ is number of items $(n=4)$ and $C$ is the total capacity $(C=5)$. Each item (i) has a knapsack weight $\left(W_{i}\right)$, and a knapsack profit $\left(P_{i}\right)$ obtained by allocating required resource to the specified item i. All $P_{i}$ s and $W_{i}$ 's are positive integer numbers.

Table 1. The expected value to activates.

$$
L=\left[\begin{array}{cccccccccccc}
0 & 5 & 6.5 & 7.5 & 0 & 0 & 0 & 0 & 0 & 0 & 0 & 0 \\
5 & 0 & 5 & 0 & 7 & 4.5 & 0 & 9 & 0 & 0 & 0 & 0 \\
6.5 & 5 & 0 & 3 & 0 & 10.5 & 0 & 0 & 0 & 0 & 0 & 0 \\
7.5 & 0 & 3 & 0 & 0 & 6.5 & 8.5 & 0 & 0 & 0 & 0 & 0 \\
0 & 7 & 0 & 0 & 0 & 6 & 0 & 7.5 & 0 & 6 & 0 & 8 \\
0 & 4.5 & 10.5 & 6.5 & 6 & 0 & 5 & 3 & 4 & 8 & 0 & 0 \\
0 & 0 & 0 & 8.5 & 0 & 5 & 0 & 0 & 5.5 & 0 & 0 & 0 \\
0 & 9 & 0 & 0 & 7.5 & 3 & 0 & 0 & 9.5 & 3.5 & 0 & 0 \\
0 & 0 & 0 & 0 & 0 & 4 & 5.5 & 9.5 & 0 & 6 & 5 & 0 \\
0 & 0 & 0 & 0 & 6 & 8 & 0 & 3.5 & 6 & 0 & 0 & 4.5 \\
0 & 0 & 0 & 0 & 0 & 0 & 0 & 0 & 0 & 5 & 0 & 7.5 \\
0 & 0 & 0 & 0 & 8 & 0 & 0 & 0 & 0 & 4.5 & 7.5 & 0
\end{array}\right]
$$

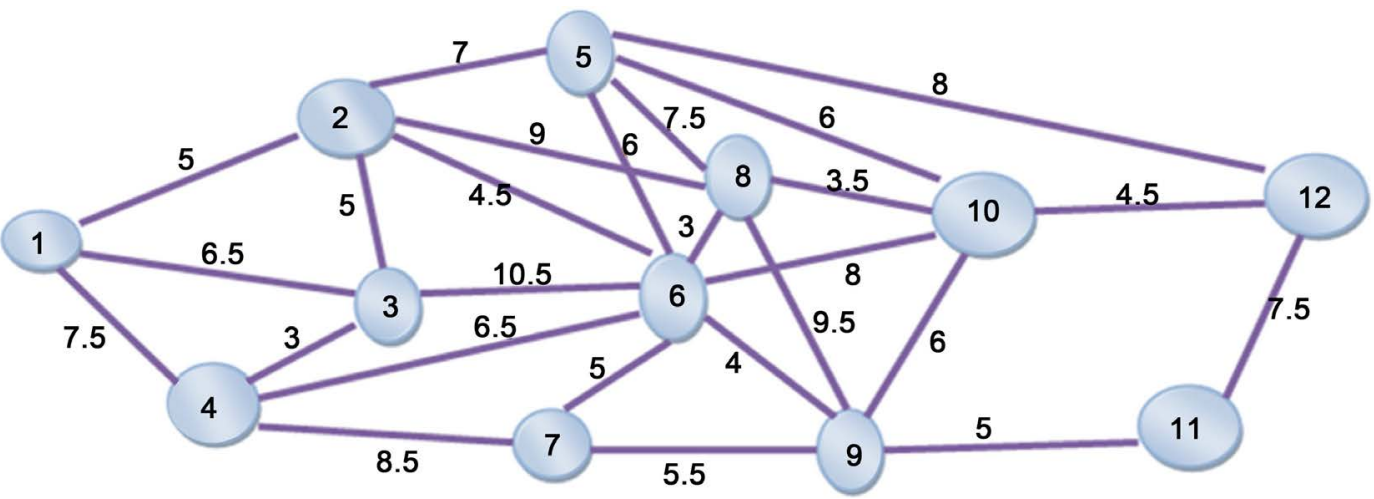

Figure 3. The converted undirected graph with 12 nodes. 
Table 2. The shortest path to network in Figure $3(1) \rightarrow(2) \rightarrow(5) \rightarrow(12)$.

\begin{tabular}{ccccccccccc}
\hline 0 & 1.9929 & 0.0012 & 0 & 0 & 1.9929 & 0 & 0 & 0 & 0 & 0 \\
0.001 & 0 & 0.001 & 0 & 1.9839 & 0 & 0 & 0.0011 & 0 & 0 & 0 \\
0.001 & 0.001 & 0 & 0.001 & 0 & 0.0012 & 0 & 0 & 0 & 0 & 0 \\
0.001 & 0 & 0.001 & 0 & 0 & 0.0011 & 0.0011 & 0 & 0 & 0 & 2.0141 \\
0 & 0.001 & 0 & 0 & 0 & 0.001 & 0 & 0.001 & 0.001 & 0 & 0 \\
0 & 0.001 & 0.001 & 0.001 & 0.001 & 0 & 0.001 & 0.0014 & 0.001 & 0 & 0 \\
0 & 0 & 0 & 0.001 & 0 & 0.0012 & 0 & 0 & 0 & 0 & 0 \\
0 & 0.001 & 0 & 0 & 0.001 & 0.001 & 0 & 0 & 0.0027 & 0 & 0 \\
0 & 0 & 0 & 0 & 0 & 0.001 & 0.001 & 0.001 & 0.001 & 0.0012 & 0 \\
0 & 0 & 0 & 0 & 0.001 & 0.001 & 0 & 0.001 & 0 & 0 & 0.0106 \\
0 & 0 & 0 & 0 & 0 & 0 & 0 & 0 & 0.001 & 0 & 0.0011 \\
0 & 0 & 0 & 0 & 0.001 & 0 & 0 & 0 & 0.001 & 0.001 & 0 \\
\hline
\end{tabular}

Then, the subproblem SP[TN, Capcount] in algorithm 4 will be computed to find optimal solution for the list (SP) of the $\mathrm{n}$ items.

The network in the formulation has several layers of nodes: It has one layer corresponding to each item and one layer corresponding to a source node $s$ and another corresponding to a sink node t. The layer corresponding to an item $i$ has $W+1$ nodes, $i^{0}, i^{1}, \cdots, i^{w}$. Node.

The knapsack problem assuming that the knapsack has a capacity of $W=6, v_{j}$ is the value of item $j, w_{j}$ is the weight of item $j$. Figure 4 can be obtained. The path in this graph corresponds the feasible solution of the knapsack problem. Node $\mathrm{S}$ means the start node, node $\mathrm{E}$ means the end node. The $1^{\text {st }}$ number in the residual circles means the item's category, the $2^{\text {nd }}$ number in these residual circles states the capability of the knapsack that the solution has consumed. The number along the arc funds the value of the consistent item. For example the circle with rate $(1,4)$ in the first layer means that the item has used 4 units of the knapsack's capacity. At the similar time, each path from node $S$ to node $E$ explains a possible answer to the problem. For example, the path $S-(1,4)-(2,6)$ - $(3,6)-(4,6)$ - EE means that item 1 and item 2 are involved in the knapsack, item 3 and item 4 are omitted. It also shows that the response to the knapsack problem match the longest track in the network.

\section{Conclusion}

The shortest path problem shows a substantial role in many usages. In this paper, based on an amoeboid creature algorithm, a new procedure is proposed to resolve the shortest path problems with interval bracket. A numeral example is explained to show the qualification of the proposed method. The 0 - 1 knapsack problem plays a substantial role in real-life applications. In this paper, based on amoeboid creature algorithm and classic 0 - 1 Knapsack Algorithm, a new method is suggested to solve classical $0-1$ knapsack problems. We have used the 


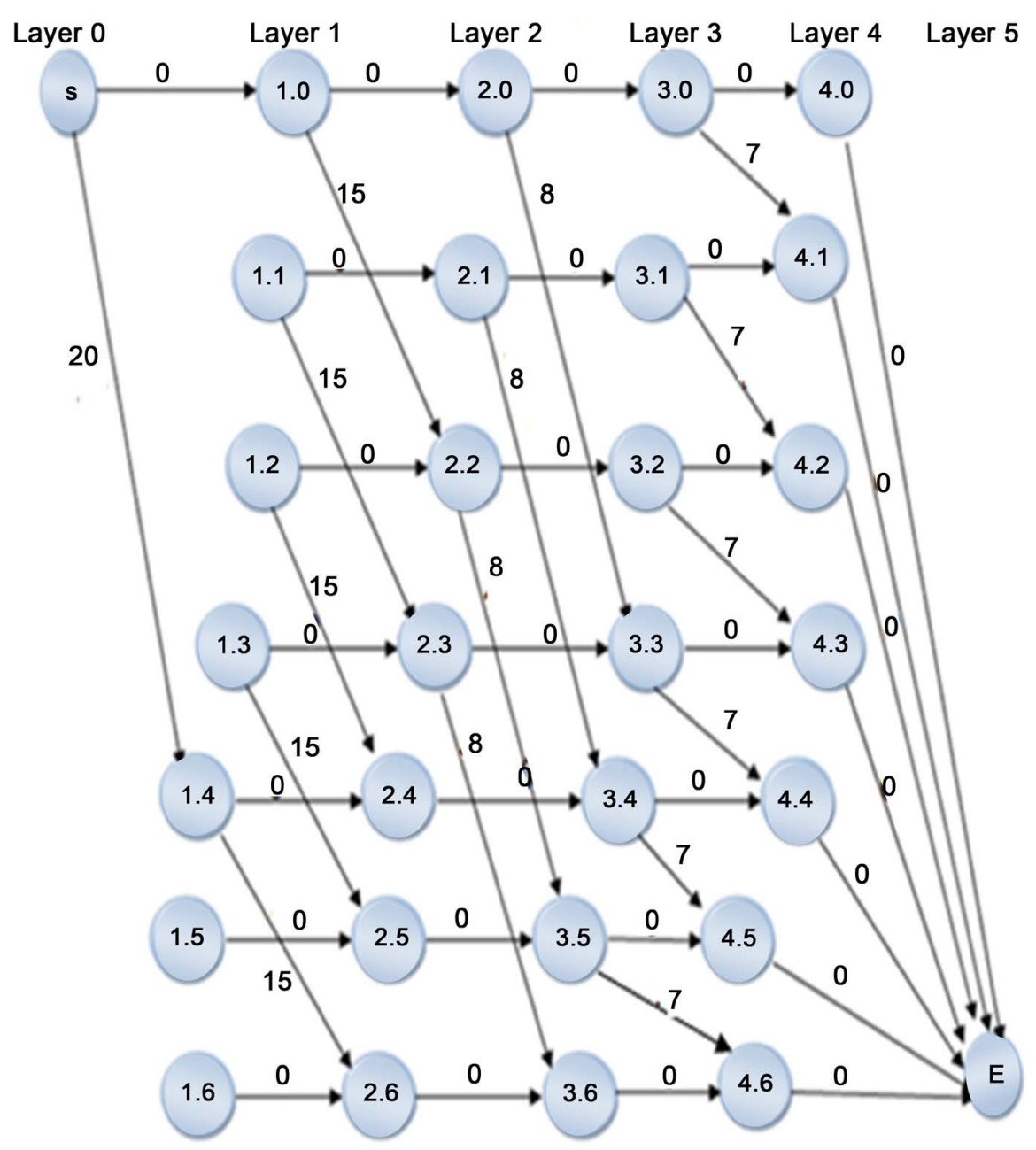

Figure 4. The longest path formulation of the knapsack problem with 4 items.

benchmark problems to exam the amoeboid creature algorithm. The computational outcomes explain the efficiency of the presented approach. One of our outstanding studies is to solve other 0 - 1 knapsack problems under additional complex situations, such as the multi-objective shortest path problem and the knapsack problem with more criteria.

\section{Conflicts of Interest}

The authors declare no conflicts of interest regarding the publication of this paper.

\section{References}

[1] Gang, Y. (1996) On the Max-Min 0-1 Knapsack Problem with Robust Optimization Applications. Journal Operations Research, 44, 407-415. https://doi.org/10.1287/opre.44.2.407

[2] Nakagaki, T., Yamada, H. and Toth, A. (2001) Path Finding by Tube Morphogenesis in an Amoeboid Organism. Biophysical Chemistry, 92, 47-52. https://doi.org/10.1016/S0301-4622(01)00179-X 
[3] Campegiani, P. and Lo Presti, F. (2009) A General Model for Virtual Machines Resources Allocation in Multitier Distributed Systems. ICAS '09. Fifth International Conference on Autonomic and Autonomous Systems, 69, 162-167.

[4] Zhang, X., Huang, Sh., Hub, Y., Zhang, Y., Mahadevan, S. and Deng, Y. (2013) Solving 0-1 Knapsack Problems Based on Amoeboid Organism Algorithm. Applied Mathematics and Computation, 219, 9959-9970.

https://doi.org/10.1016/j.amc.2013.04.023

[5] Robert, R.P. and Van Heerde, H.J. (2016) Robust Optimization of the 0-1 Knapsack Problem: Balancing Risk and Return in Assortment Optimization. European Journal of Operational Research, 250, 842-854. https://doi.org/10.1016/j.ejor.2015.10.014

[6] Tero, A., Kobayashi, R. and Nakagaki, T. (2007) A Mathematical Model for Adaptive Transport Network in Path Finding by True Slime Mold. Journal of Theoretical Biology, 244, 553-564. https://doi.org/10.1016/j.jtbi.2006.07.015

[7] Ahuja, R., Magnanti, T., Orlin, J. and Weihe, K. (1995) Network Flows: Theory, Algorithms and Applications. ZOR - Mathematical Methods of Operations Research, 41, 252-254.

[8] Zhang, X.G., Zhang, Y.J. and Wei, D.J. (2012) Solving Shortest Path Problems with Interval Arcs Based on an Amoeboid Organism Algorithm. Journal of Information \& Computational Science, 9, 2081-2088. http://www.joics.com

[9] Zhang, G.J., Yang, H.J. and Liu, Z. (2007) Using Watering Algorithm to Find the Optimal Paths of a Maze. Computer Simulation, 24, 171-173.

[10] Nakagaki, T., Yamada, H. and Tóth, A., et al. (2000) Maze-Solving by an Amoeboid Organism. Nature, 407, 470-471. https://doi.org/10.1038/35035159

[11] Nakagaki, T., Yamada, H. and Hara, M. (2004) Smart Network Solutions in an Amoeboid Organism. Biophysical Chemistry, 107, 1-5.

https://doi.org/10.1016/S0301-4622(03)00189-3

[12] Deng, Y., Jiang, W. and Sadiq, R. (2011) Modelling Contaminant Intrusion in Water Distribution Networks: A New Similarity-Based DST Method. Expert Systems with Applications, 38, 571-578. https://doi.org/10.1016/j.eswa.2010.07.004 